Article

\title{
Physical Parameterization of Hyperspectral Reflectance in the Oxygen A-Band for Single-Layer Water Clouds
}

\author{
Jie Yang ${ }^{1}$, Siwei Li ${ }^{1,2, *}$, Feiyue Mao ${ }^{2}$, Qilong Min ${ }^{3}$, Wei Gong ${ }^{1}$, Lei Zhang ${ }^{2}$ and Sheng Liu ${ }^{2}$ \\ 1 State Key Laboratory of Information Engineering in Surveying, Mapping and Remote Sensing, \\ Wuhan University, Wuhan 430079, China; jie.yang@whu.edu.cn (J.Y.); weigong@whu.edu.cn (W.G.) \\ 2 School of Remote Sensing and Information Engineering, Wuhan University, Wuhan 430079, China; \\ maofeiyue@whu.edu.cn (F.M.); whuygzhanglei@whu.edu.cn (L.Z.); shengliu-ars@whu.edu.cn (S.L.) \\ 3 Atmospheric Sciences Research Center, The State University of New York at Albany, Albany, NY 12222, USA; \\ qmin@albany.edu \\ * Correspondence: siwei.li@whu.edu.cn
}

Received: 12 June 2020; Accepted: 8 July 2020; Published: 14 July 2020

\begin{abstract}
Previous studies have shown that it is feasible to retrieve multiple cloud properties simultaneously based on the space-borne hyperspectral observation in the oxygen A-band, such as cloud optical depth, cloud-top height, and cloud geometrical thickness. However, hyperspectral remote sensing is time-consuming if based on the precise radiative transfer solution that counts multiple scatterings of light. To speed up the radiation transfer solution in cloud scenarios for nadir space-borne observations, we developed a physical parameterization of hyperspectral reflectance in the oxygen A-band for single-layer water clouds. The parameterization takes into account the influences of cloud droplet forward-scattering and nonlinear oxygen absorption on hyperspectral reflectance, which are improvements over the previous studies. The performance of the parameterization is estimated through comparison with DISORT (Discrete Ordinates Radiative Transfer Program Multi-Layered Plane-Parallel Medium) on the cases with solar zenith angle $\theta$, the cloud optical depth $\tau_{c}$, and the single-scattering albedo $\omega$ in the range of $0 \leq \theta \leq 75,5 \leq \tau_{c} \leq 50,0.5 \leq \omega \leq 1$. The relative error of the cloud reflectance is within $5 \%$ for most cases, even for clouds with optical depths around five or at strong absorption wavelengths. We integrate the parameterization with a slit function and a simplified atmosphere to evaluate its performance in simulating the observed cloud reflection at the top of the atmosphere by OCO-2 (Orbiting Carbon Observatory-2). To better visualize the possible errors from the new parameterization, gas molecular scattering, aerosol scattering, and reflection from the underlying surface are ignored. The relative error of the out-of-band radiance is less than $4 \%$ and the relative error of the intra-band radiance ratio is less than $4 \%$. The radiance ratio is the ratio of simulated observations with and without in-cloud absorption and is used to assess the accuracy of the parameterization in quantifying the in-cloud absorption. The parameterization is a preparation for rapid hyperspectral remote sensing in the oxygen A-band. It would help to improve retrieval efficiency and provide cloud geometric thickness products.
\end{abstract}

Keywords: clouds; oxygen A-band; radiative transfer

\section{Introduction}

Inferring cloud geometrical properties from the space-borne observation in the oxygen A-band has been recognized for a long time [1]. The principle is that cloud scattering affects the photon path, and the photon path length determines the total oxygen absorption along the path. The cloud-top height (CTH) and the cloud geometrical thickness (CGT) are important factors affecting the photon 
paths above and below the cloud top, respectively. Therefore, the key to retrieve CTH and CGT is to accurately distinguish and calculate the oxygen absorption above and inside clouds [2]. Besides, an accurate calculation of the radiance that is not interfered by oxygen absorption helps to retrieve the cloud optical depth (COD).

Several algorithms have been proposed for cloud retrieval in the oxygen A-band, such as FRESCO (Fast Retrieval Scheme for Clouds from the Oxygen A-band) [3], SACURA (Semi-Analytical Cloud Retrieval Algorithm) [4], and ROCINN (Retrieval of Cloud Information using Neural Networks [5,6]). They are developed for space-borne sensors with coarse footprint size (larger than $30 \mathrm{~km} \times 60 \mathrm{~km}$ ) and moderate spectral resolution (about 0.33-0.5 nm), including GOME (Global Ozone Monitoring Experiment) [7], SCIAMACHY (Scanning Imaging Absorption Spectrometer for Atmospheric Cartography) [8-10] and GOME-2 [11-15]. An essential difference between these algorithms is the parameterization of the anisotropic cloud-top reflectance, which are either based on the "Clouds-as-Reflecting-Boundaries" (CRB) model or the "Clouds-As-Layers" (CAL) model [6]. The CRB model (e.g., FRESCO), treats the cloud as an opaque slab, taking the cloud-top height and the cloud albedo as parameters. The CAL model (e.g., SACURA), regards the cloud as a medium composed of scattering particles and takes cloud-top height and cloud optical depth as parameters. The real reflectance is related not only to the cloud optical depth but also to the cloud structure characteristics, such as the cloud geometrical thickness and the multiple cloud layers [16]. The retrieved CTH will be biased if differences in in-cloud absorption due to cloud structure are not accurately quantified [17,18].

It is challenging to allow for the influence of cloud structure on medium spectral resolution observations in retrievals, which contains not enough information [19]. SACURA considers the in-cloud scattering and absorption in the cloud reflectance calculation, and reduces the uncertainty of CTH retrievals when CGT was estimated from independent observations [20], or retrieves CTH and CGT simultaneously. The error of simultaneously retrieved CGT is generally larger than that of CTH-up to three times in the case of a single-layer water cloud [4].

In contrast, hyperspectral measurements can provide more accurate CTH [21], and have the potential to estimate CGT to within $10 \%$ with little reliance on any prior data due to the wide range of oxygen absorption strength [22]. In recent years, sensors with hyperspectral resolution (about $0.03-0.044 \mathrm{~nm}$ ) in the oxygen A-band have been developed and launched with satellites, such as GOSAT (Greenhouse gas Observing Satellite) [23] and OCO-2 (Orbiting Carbon Observatory-2) [24]. The information content of OCO-2 A-band measurements is sufficient to allow for the simultaneous retrievals of COD, CTH, and CGT $[25,26]$.

However, hyperspectral remote sensing is time-consuming, if based on the precise radiative transfer solution counting multiple scatterings of light. To decrease the computational burden without compromising accuracy, Maximilian Reuter et al. propose the FOCAL (Fast atmospheric trace gas retrieval) for CO2 retrievals in scenarios where scattering in clouds is minimal [27], and Ana del Águila et al. propose the CLSR method (Cluster Low-Streams Regression), based on the strong close-to-linear relationship between the low- and multi-stream model [28]. For rapid cloud retrievals, we are mainly interested in cloud scattering, and a model that can be directly differentiable to cloud properties. SACURA is one such algorithm that uses a differentiable formula, rather than a time-consuming model to calculate the cloud reflectance [29], although it is only appliable for the GOME type observations consisting only of non-absorption and weak absorption channels (single-scattering albedo is higher than 0.95) [30]. The error increases when applying to hyperspectral simulation, which is more sensitive to the oxygen absorption lines, as shown in Figure 1. The approximation formula will expand to strong absorption wavelengths if a lookup table is built to replace the part of the formula that is not suitable for lower single-scattering albedo [31]. As a side effect of using lookup tables, the approximation formula will lose the advantage of being directly differentiable, which is another reason why it is faster than other numerical solutions. 


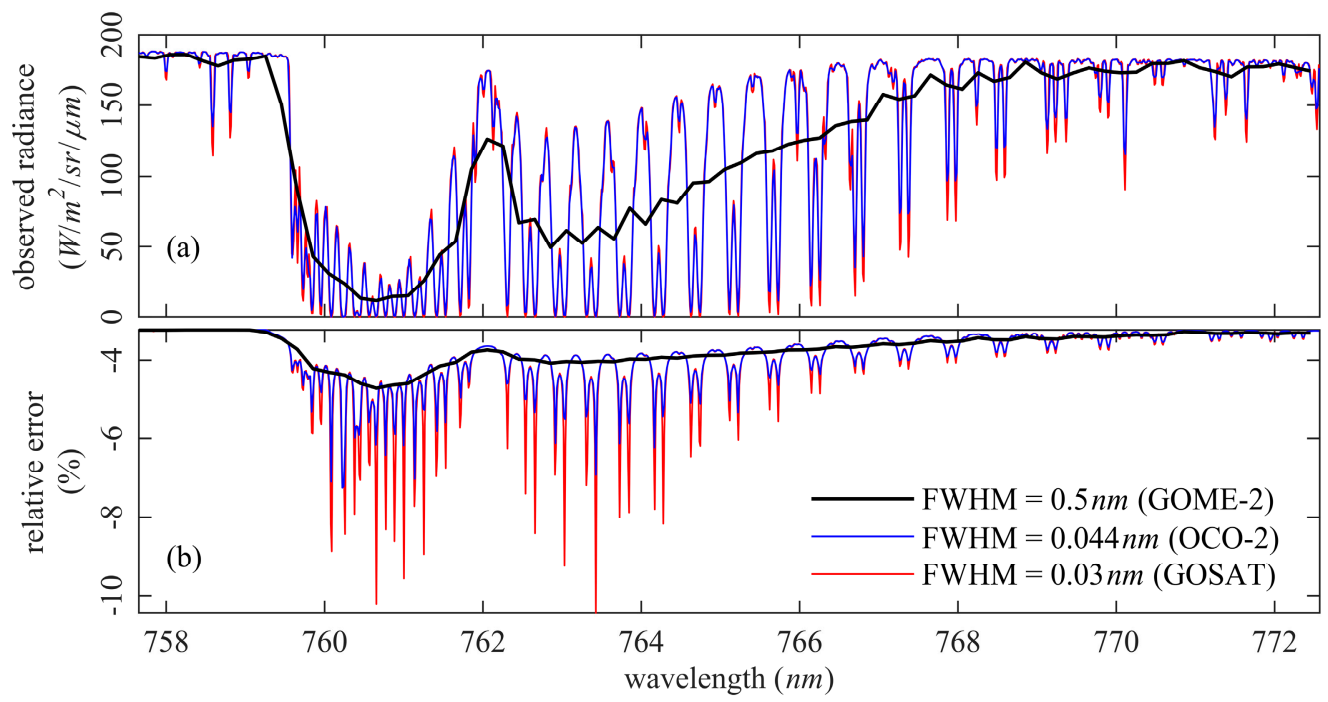

Figure 1. (a) Precise top-of-atmospheric radiance simulated by DISORT (Discrete Ordinates Radiative Transfer Program for a Multi-Layered Plane-Parallel Medium) with different spectral resolution full width at half maximum (FWHM). (b) The relative error between the precise radiance and the radiance calculated according to the previous study [32]. The observations of OCO-2 and GOSAT have sharp and deep absorption channels, while those of GOME-2 do not. When applying the previous formula [32] to hyperspectral simulation, the relative error is doubled. The sampling number per FWHM is 2.5. The cloud optical depth used in the simulation is 15 , the cloud top height is $2 \mathrm{~km}$, the cloud geometrical thickness is $1 \mathrm{~km}$, and the solar zenith angle is $40^{\circ}$.

For fast and accurate hyperspectral remote sensing, we established a parameterization of the cloud reflectance that applies to both weak and strong absorption wavelengths. The parameterization consists of several functions with physical meaning, which reproduce the process of radiation transfer in the cloud. In the next section, we will explain the in-cloud scattering and absorption difference between weak and strong absorption situations. Then, we present the improved parameterization and a simplified atmosphere model used in accuracy evaluation. We verify the necessity of the improvements and estimate the accuracy of the parameterized cloud reflectance and the simulated hyperspectral radiance in Section 3. Section 4 discusses the source of errors, deficiencies, and future research directions and summarize the improvements and the performance of the physical parameterization.

\section{Methodology}

The physical parameterization originates from the absorption-free semi-infinite cloud case. The absorption refers to the absorption of oxygen molecules, as the absorption of water vapor and cloud droplets is far less than that of oxygen in the A-band and ignored in cloud retrievals. Therefore, the out-of-band absorption optical depth is regarded as 0 , and the single scattering albedo of the cloud layer is 1 . In the asymptotic theory, the cloud-top reflectance $R_{\infty}^{0}$ can be parameterized by the viewing geometry [33,34]. The superscript $(0)$ of $R_{\infty}^{0}$ means no absorption in the cloud layer, and the subscript $(\infty)$ means infinite cloud optical depth. Oxygen molecules absorb solar radiation in the A-band, reducing the cloud reflectance $[35,36]$. With the function $S$ representing the attenuation, due to absorption by oxygen, the reflectance $R_{\infty}$ of the semi-infinite cloud could be expressed as:

$$
R_{\infty}=R_{\infty}^{0} S .
$$


Since most real-world clouds are optically limited, part of the solar radiation penetrates clouds and further reduces the reflectance [30]. With the function $H$ standing for the loss due to penetration, the cloud reflectance $R$ can be represented as:

$$
R=R_{\infty}-H
$$

Kokhanovsky et al. divide $R_{\infty}^{0}$ into a single-scattering part $\left(R_{\infty, \mathrm{ss}}^{0}\right)$, and a multi-scattering part $\left(R_{\infty, \mathrm{ms}}^{0}\right)$, depending on whether related to the cloud phase function directly [30,32,34,37]. They also propose another formula for $R_{\infty}^{0}$ in SACURA [4,33], where the phase-function-dependent part would vanish for nadir observations. In this study, we focused on nadir observations but considered cloud phase function, so the cloud reflectance, based on [30,34,37], could be written as:

$$
R=\left(R_{\infty, \mathrm{ss}}^{0}+R_{\infty, \mathrm{ms}}^{0}\right) S-H
$$

Dividing the reflectance into single- and multi-scattering parts could better explain how the cloud reflectance is related to the viewing geometry. Still, the same functions $S$ and $H$ are used in both parts. For weak absorption, the single-scattering part accounts for a small proportion in cloud reflection, as shown in Figure $2 \mathrm{a}$, and hence it is unnecessary to define exclusive functions for $R_{\infty, \mathrm{ss}}^{0}$. However, as the absorption increases, it is inadequate to use one function $S$ for the two parts with different in-cloud absorption attenuation, as shown in Figure $2 b$. Similar, different $H$ functions are also needed for the two parts.
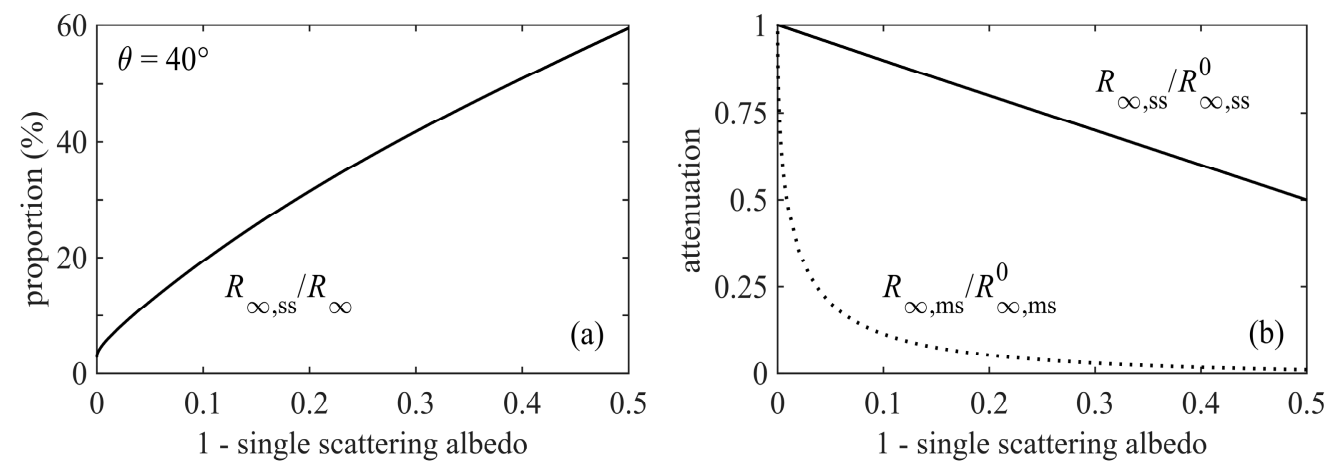

Figure 2. (a) The proportion of the single-scattering part increases during absorption enhancement. (b) The curve of absorption attenuation is very different between the single- and multi-scattering parts. The single-scattering part $\left(R_{\infty, \mathrm{ss}}^{0}, R_{\infty, \mathrm{ss}}\right)$ was calculated according to the definition of single-scattering. The cloud reflectance $\left(R_{\infty}^{0}, R_{\infty}\right)$ was calculated by DISORT. The multi-scattering part $\left(R_{\infty, \mathrm{ms}}^{0}, R_{\infty, \mathrm{ms}}\right)$ is the difference between the cloud reflectance and the single-scattering part.

Moreover, forward-scattering impacts should be well resolved, since it retains direct-light-like directivity, which will enhance the radiance in the incidence direction, particularly crucial for optically thin clouds because of less multi-scattering. Considering that the single-scattering part is closely related to the cloud phase function, the phase-function-dependent part, $R_{\mathrm{ph}}$, is the result of the scatterings of direct solar radiation and the forward-scattered radiance. The other part, $R_{\mathrm{ms}}$, is the reflectance that does not depend directly on the cloud phase function because of the randomizing effect of multi-scattering.

Our work consisted of two parts: quantifying the contribution of the forward-scattering to $R_{\mathrm{ph}}$ and modifying $R_{\mathrm{ms}}$ to handle the enhanced absorption. The physical parameterization expressed the reflectance $(R)$ in terms of the attenuation due to absorption $\left(S_{\mathrm{ph}}, S_{\mathrm{ms}}\right)$ and loss due to penetration $\left(H_{\mathrm{ph}}, H_{\mathrm{ms}}\right)$ as:

$$
\begin{gathered}
R=R_{\mathrm{ph}}+R_{\mathrm{ms}}, \\
R_{\mathrm{ph}}=R_{\infty, \mathrm{ph}}-H_{\mathrm{ph}},
\end{gathered}
$$




$$
\begin{gathered}
R_{\mathrm{ms}}=R_{\infty, \mathrm{ms}}-H_{\mathrm{ms}}, \\
R_{\infty, \mathrm{ph}}=R_{\infty, \mathrm{ph}}^{0} S_{\mathrm{ph}} \\
R_{\infty, \mathrm{ms}}=R_{\infty, \mathrm{ms}}^{0} S_{\mathrm{ms}} .
\end{gathered}
$$

Three types of clouds are involved in the above formulas and the reflectance of each type contains parts that are dependent and independent of the phase function. Except for the fact that the sum of $R_{\mathrm{ph}}$ and $R_{\mathrm{ms}}$ is the reflectance of the real-world cloud, $R_{\infty, \mathrm{ph}}$ and $R_{\infty, \mathrm{ms}}$ correspond to the reflectance of the semi-infinite cloud $\left(R_{\infty}\right)$, and $R_{\infty, \mathrm{ph}}^{0}$ and $R_{\infty, \mathrm{ms}}^{0}$ correspond to the reflectance of the absorption-free semi-infinite cloud $\left(R_{\infty}^{0}\right)$.

$$
\begin{aligned}
& R_{\infty}=R_{\infty, \mathrm{ph}}+R_{\infty, \mathrm{ms}} \\
& R_{\infty}^{0}=R_{\infty, \mathrm{ph}}^{0}+R_{\infty, \mathrm{ms}}^{0} .
\end{aligned}
$$

\subsection{Phase-Function-Dependent Reflectance}

Based on the definition of reflectance, $R_{\mathrm{ph}}$ for the nadir observation is:

$$
\begin{gathered}
R_{\mathrm{ph}}(\mu, \omega, \tau)=\frac{\int_{0}^{\tau} J_{\mathrm{ph}}\left(\theta, \omega, \tau^{\prime}, \pi-\theta\right) \exp \left(-\tau^{\prime}\right) d \tau^{\prime}}{F \mu / \pi}, \\
J_{\mathrm{ph}}\left(\theta, \omega, \tau^{\prime}, \vartheta\right)=\sum_{i=0}^{\infty} J_{i}\left(\theta, \omega, \tau^{\prime}, \vartheta\right),
\end{gathered}
$$

where $F$ is the solar radiation at the cloud top, and $\mu$ is the cosine of solar zenith angle $\theta . \tau$ and $\omega$ are the extinction optical depth and the single-scattering albedo of the cloud layer, respectively. The phase-function-dependent source function, $J_{\mathrm{ph}}$, consists of contributions from direct solar radiation $\left(J_{1}\right)$ and forward-scattered radiance $\left(J_{2}\right.$ to $\left.J_{n}\right)$. The subscript $n$ of $J_{n}$ indicates that the radiation source has previously scattered forward $(n-1)$ times. Each forward-scattered radiance has the same direction as direct solar radiation.

For the single-scattering of direct solar radiation, the source function $J_{1}$ can be written as:

$$
J_{1}\left(\theta, \omega, \tau^{\prime}, \vartheta\right)=F \exp \left(-\frac{\tau^{\prime}}{\mu}\right) \times \frac{\omega p(\vartheta)}{4 \pi}
$$

where $F \exp \left(-\tau^{\prime} / \mu\right)$ is the attenuated solar radiation at the optical depth $\tau^{\prime}, p(\vartheta)$ is the water cloud phase function, and $\vartheta$ is the scattering angle. For the nadir observation, $\vartheta=\pi-\theta$. For the forward-scattering, $\vartheta=0^{\circ}$.

The source function $\mathrm{J}_{2}$ stands for the single-scattering of the first forward-scattered radiance:

$$
J_{2}\left(\theta, \omega, \tau^{\prime}, \vartheta\right)=\int_{0}^{\tau^{\prime}} J_{1}\left(\theta, \omega, \tau^{\prime \prime}, 0\right) \exp \left(-\frac{\tau^{\prime}-\tau^{\prime \prime}}{\mu}\right) d \tau^{\prime \prime} \times \frac{\omega p(\vartheta)}{4 \pi}
$$

The integral in Equation (14) is the sum of all the first forward-scattered radiance reaching the extinction optical depth $\tau^{\prime}$. Simplifying the above formula, we have:

$$
J_{2}\left(\theta, \omega, \tau^{\prime}, \vartheta\right)=J_{1}\left(\theta, \omega, \tau^{\prime}, \vartheta\right) \tau^{\prime} \omega p(\vartheta) / 4 \pi
$$

Similarly, the source function $J_{n}$ formed by the $(n-1)$-times-forward-scattered radiance is:

$$
J_{n}\left(\theta, \omega, \tau^{\prime}, \vartheta\right)=J_{n-1}\left(\theta, \omega, \tau^{\prime}, \vartheta\right) \frac{\tau^{\prime}}{n-1} \omega p(\vartheta) / 4 \pi
$$


Substituting Equation (16) into Equation (12), the source function that depends on the phase function is:

$$
J_{\mathrm{ph}}\left(\theta, \omega, \tau^{\prime}, \vartheta\right)=F \exp \left(-\frac{\tau^{\prime}}{\mu}+\frac{\tau^{\prime} \omega p(0)}{4 \pi}\right) \times \frac{\omega p(\vartheta)}{4 \pi} .
$$

Compared with Equation (13), the additional positive term $\tau^{\prime} \omega p(0) / 4 \pi$ in Equation (17) means that, except for the attenuated sunlight, forward-scattered radiance also contributes to $R_{\text {ph }}$. The factor $\exp \left[\tau^{\prime} \omega p(0) / 4 \pi\right]$ only counts the forward-scattered radiance with a scattering angle of $0^{\circ}$, and the scattered radiance, which has small scattering angles, also contributes to $J_{\mathrm{ph}}$. So, we replaced $p(0) / 4 \pi$ with an undetermined coefficient $c$ to take in more sources that give rise to the phase-function-dependent reflectance. Substituting Equation (17) into Equation (11), each term of $R_{\mathrm{ph}}$ for nadir observation can be expressed as:

$$
\begin{gathered}
R_{\infty, \mathrm{ph}}^{0}(\mu)=p(\pi-\theta) / 4 /(\mu+1-c \mu), \\
S_{\mathrm{ph}}(\mu, \omega)=\omega(\mu+1-c \mu) /(\mu+1-c \mu \omega), \\
H_{\mathrm{ph}}(\mu, \omega, \tau)=\exp (-\tau / \mu+\tau \omega c)\left(R_{\infty, \mathrm{ph}}^{0} S_{\mathrm{ph}}\right) \exp (-\tau) .
\end{gathered}
$$

In the derivation, the nothingness beneath the optically finite cloud $L$ can be thought of as a disappeared semi-infinite cloud $L_{\infty}$. It has the same single-scattering albedo with $L$. If $L_{\infty}$ were still there, the reflection from the top of $L$ would be brighter than if it was only $L$. The cloud-top reflection reduces with the disappearance of $L_{\infty}$. The three terms in $H_{\text {ph }}$ accurately describe the penetration loss, which penetrates $L$ from top to bottom, reflected by $L_{\infty}$, and finally passes through $L$ again from the bottom up. After $L_{\infty}$ disappears, this radiation cannot return to the cloud top and should be subtracted.

\subsection{Phase-Function-Independent Reflectance}

The phase-function-independent reflectance is composed of $R_{\infty, \mathrm{ms}}^{0}$ related to the viewing geometry, $S_{\mathrm{ms}}$ representing absorption, and $H_{\mathrm{ms}}$ standing for the penetration loss. Multi-scattering makes $R_{\infty, \mathrm{ms}}^{0}$ less dependent on the phase function. A rational polynomial would well fit the reflectance changed with the viewing geometry:

$$
R_{\infty, \mathrm{ms}}^{0}(\mu)=\left(d_{0}+d_{1} \mu\right) /\left(1+d_{2} \mu\right) .
$$

The coefficients $d_{0}, d_{1}$, and $d_{2}$ are determined in Section 2.3.

SACURA uses a parameter $y$ to play the role of absorption in the radiative transfer of diffused light [37]. With absorption enhancement, high-order terms are needed to ensure accuracy [35]. After adding the fourth-order polynomial with nine undetermined coefficients $\left(e_{00}, e_{10}\right.$, etc.), the improved function $S_{\mathrm{ms}}$ applicable to absorption lines could be expressed as:

$$
\begin{gathered}
S_{\mathrm{ms}}(\mu, \omega)=\exp \left[-y\left(e_{00}+e_{10} \mu+e_{01} y+e_{11} \mu y+e_{02} y^{2}+e_{12} \mu y^{2}+e_{03} y^{3}+e_{13} \mu y^{3}+e_{04} y^{4}\right)\right], \\
y=4 \sqrt{3(1-\omega)(1-\omega g)} /[3(1-g)] .
\end{gathered}
$$

The function $H_{\mathrm{ms}}$, similar to $H_{\mathrm{ph}}$, consists of the three processes: (a) penetrating through $L$, (b) reflected by $L_{\infty}$, and (c) penetrating $L$ again. SACURA gives each process a parameterization when $\omega>0.95$ :

1. The global transmittance $t$ defines the first penetration. Besides, a correction $\Delta t$ is proposed for optically relatively thin clouds [30].

2. The diffuse reflection of $L_{\infty}$ is quantified as a spherical albedo and approximately equals to $\exp (-y)[38]$.

3. When the diffused light penetrates $L$ from the bottom up, the attenuation is similar to the radiative transfer in the optically deep region, which is approximated by $\exp (-x)$ [37]. 
Since the parameterizations $(t, \Delta t, \exp (-y)$, and $\exp (-x))$ are integrals of the diffuse radiation over incidence and exit directions, the asymptotic theory uses the escape function $K$ to deal with the viewing geometry. The function $H_{\mathrm{ms}}$ in SACURA is [30]:

$$
\begin{gathered}
H_{\mathrm{ms}}(\mu, \omega, \tau)=K(\mu, \omega)[t(\omega, \tau)-\Delta t(\mu, \omega, \tau) \exp (x)] \times \exp (-y) \exp (-x) K(1, \omega), \\
x=\tau \sqrt{3(1-\omega)(1-\omega g)} .
\end{gathered}
$$

For cases with strong absorptions (i.e., $\omega<0.95)$, we made modifications so that each part of the function $H_{\mathrm{ms}}$ was close to the numerical solution of the radiative transfer equation, such as adding $\exp \left[x\left(m_{0}+m_{1} y+m_{2} y^{2}\right)\right], y\left(p_{2}+p_{3} \mu+p_{4} \mu^{2}\right)$, and $\left(1+q_{3} y\right)$ into the functions $t, K$, and $\Delta t$, respectively. The improved formulas are:

$$
\begin{gathered}
t(\omega, \tau)=\frac{\sinh (y)}{\sinh (x+\alpha y)} \times \exp \left[x\left(m_{0}+m_{1} y+m_{2} y^{2}\right)\right], \\
K(\mu, \omega)=\left(p_{0}+p_{1} \mu\right)+y\left(p_{2}+p_{3} \mu+p_{4} \mu^{2}\right), \\
\Delta t(\mu, \omega, \tau)=\left(q_{0}+q_{1} \mu+q_{2} \mu^{2}\right) /\left(\tau^{3} \omega^{3}\right) \times\left(1+q_{3} y\right) .
\end{gathered}
$$

The modifications would not affect the applicability to weak absorption, because of the degeneration from the improved formulas to the previous when $y \rightarrow 0$. The coefficients $m_{0}, p_{0}$, $q_{0}$, etc. are determined in Section 2.3.

\subsection{Coefficient Fitting}

The improved formulas make up the physical parameterization for single-layer water clouds:

$$
\begin{gathered}
R_{\infty}(\mu, \omega)=R_{\infty, \mathrm{ph}}^{0}(\mu) S_{\mathrm{ph}}(\omega)+R_{\infty, \mathrm{ms}}^{0}(\mu) S_{\mathrm{ms}}(\mu, \omega), \\
R(\mu, \omega, \tau)=R_{\infty}(\mu, \omega)-H_{\mathrm{ph}}(\mu, \omega, \tau)+H_{\mathrm{ms}}(\mu, \omega, \tau) .
\end{gathered}
$$

As the reflectance $R_{\infty}$ is a part of $R$, the coefficients in Equations (29) and (30) should be fitted in two steps. In the search for the best coefficients, the relative errors of the parameterization were used as the fitting cost, whose mean square should descend in iterations. However, it is not appropriate to take the relative error of $R_{\infty}$ as its own cost, because the infinite optical depth makes $R_{\infty}$ much larger than $R$, the error of the first fitting would destroy the second one. To solve the problem, we used the reflectance of low-level stratocumulus clouds over the ocean as the denominator of the cost in the first step, which had a statistically average cloud optical depth of 7 [39]. The cost functions $\delta_{1}$ and $\delta_{2}$ for the two-step fitting are:

$$
\begin{gathered}
\delta_{1}(\mu, \omega)=\left[R_{\infty}(\mu, \omega)-\widetilde{R}_{\infty}(\mu, \omega)\right] / \widetilde{R}(\mu, \omega, \tau=7 / \omega), \\
\delta_{2}(\mu, \omega, \tau)=[R(\mu, \omega, \tau)-\widetilde{R}(\mu, \omega, \tau)] / \widetilde{R}(\mu, \omega, \tau),
\end{gathered}
$$

where the truth reflectance $\widetilde{R}_{\infty}$ and $\widetilde{R}$ were derived from DISORT (Discrete Ordinates Radiative Transfer Program for a Multi-Layered Plane-Parallel Medium) [40] with the assumption of cloud C1 phase function. The coefficient fitting was conducted on the cases with solar zenith angle $\theta$, the cloud optical depth $\tau_{\mathcal{c}}$, and the single-scattering albedo $\omega$ in the range of $0 \leq \theta \leq 75,5 \leq \tau_{\mathcal{c}} \leq 50,0.5 \leq \omega \leq 1$. The fitting results are shown in Table 1. 
Table 1. The fitted coefficients in the physical parameterization for single-layer water clouds.

\begin{tabular}{clll}
\hline Function & \multicolumn{1}{c}{ Coefficient } \\
\hline$R_{\infty, \mathrm{ph}}^{0}$ & $c=1.0511$ & & \\
$R_{\infty, \mathrm{ms}}^{0}$ & $d_{0}=0.3395$ & $d_{1}=2.3560$ & $d_{2}=1.3758$ \\
$S_{\mathrm{ms}}$ & $e_{00}=1.1530$ & $e_{10}=0.3372$ & $e_{01}=-0.1288$ \\
& $e_{02}=1.1585 \times 10^{-2}$ & $e_{11}=-0.1234$ & $e_{03}=-6.1174 \times 10^{-4}$ \\
& $e_{12}=1.5450 \times 10^{-2}$ & $e_{04}=1.8371 \times 10^{-5}$ & $e_{13}=-6.4980 \times 10^{-4}$ \\
$t$ & $m_{0}=-0.1225$ & $m_{1}=0.4910$ & $m_{2}=-5.4428 \times 10^{-2}$ \\
$K$ & $p_{0}=0.4390$ & $p_{1}=0.8451$ & $p_{2}=-2.3089 \times 10^{-2}$ \\
& $p_{3}=-0.2831$ & $p_{4}=0.2662$ & \\
$\Delta t$ & $q_{0}=7.0239$ & $q_{1}=-21.9991$ & $q_{2}=20.1952$ \\
& $q_{3}=-0.5214$ & & \\
\hline
\end{tabular}

\subsection{Hyperspectral Simulation}

The hyperspectral radiance simulation reproduces the radiative transfer of solar radiation passing through the atmosphere, reflected by clouds to the top of the atmosphere (TOA), and finally captured by the instrument:

$$
I\left(\lambda, \mu, \tau_{c}, h, l\right)=\int_{\lambda-\Delta \lambda}^{\lambda+\Delta \lambda} f\left(\lambda, \lambda^{\prime}\right) F_{\odot}\left(\lambda^{\prime}\right) T_{\mathrm{inc}}\left(\lambda^{\prime}, \mu, h\right) \frac{\mu}{\pi} R\left(\lambda^{\prime}, \mu, \tau_{c}, l\right) T_{\mathrm{ref}}\left(\lambda^{\prime}, h\right) d \lambda^{\prime} .
$$

$\lambda$ is the central wavelength of a channel of the instrument, and $F_{\odot}$ is the extraterrestrial solar radiation at TOA. The simulation is a convolution of the reflected radiance at TOA and the instrument response function $f$. The cloud reflectivity is $\mu R / \pi$, whose optical depth is $\tau_{c}$ and geometrical thickness is $l$. The formula also contains the radiative transfer above clouds. The symbol $h$ is the cloud-top height. $T_{\text {inc }}$ is the transmissivity of the solar radiation from TOA to the cloud top, and $T_{\text {ref }}$ is the transmissivity from the cloud top to the space-borne sensor at the nadir observation. The simulations used the same instrument response function as the OCO-2 L2 retrievals [41,42], which are recorded in the OCO2_L1B_Science product (https://oco2.jpl.nasa.gov/oco-2-data-center/). The solar radiation is from the AER solar irradiance model (Atmospheric and Environmental Research, http://rtweb.aer.com/solar_frame.html) [43].

For estimating the parameterization in the hyperspectral application, a simplified atmosphere model was introduced in simulations, ignoring the molecular scattering, aerosol scattering, and reflection from the underlying surface. The spectral oxygen absorption coefficients came from LBLRTM (Line-By-Line Radiative Transfer Model) (Atmospheric and Environmental Research, Lexington, MA, USA) [44] with the assumption of the US 1976 standard atmosphere. To further simplify the atmosphere model, the oxygen absorption coefficients were fixed as a constant $k(\lambda)$ below $h_{0}=5 \mathrm{~km}$, which only changed with the wavelength. So, the total oxygen zenith optical depth $\left(\tau_{O 2, \text { total }}\right)$ from TOA to a certain height was a linear function of height $(h<5 \mathrm{~km})$, and the in-cloud oxygen absorption optical depth $\left(\tau_{\mathrm{O} 2}\right)$ was a linear function of $l$, too. The single-scattering albedo at each wavelength was calculated according to $\tau_{c}$ and $\tau_{\mathrm{O} 2}$ :

$$
\begin{gathered}
\tau_{O 2, \text { total }}(\lambda, h)=\tau_{O 2, \text { total }}\left(\lambda, h_{0}\right)+k(\lambda)\left(h-h_{0}\right) \\
\tau_{O 2}=\tau_{O 2, \text { total }}(\lambda, h+l)-\tau_{O 2, \text { total }}(\lambda, h)=k(\lambda) l \\
\omega=\frac{\tau_{\mathcal{c}}}{\tau_{c}+\tau_{O 2}}
\end{gathered}
$$


The transmissivities of the plane-parallel's atmosphere $T_{\text {inc }}$ and $T_{\text {ref }}$ depend on the slant path and $\tau_{\mathrm{O} \text {,total }}$. For nadir observation:

$$
\begin{gathered}
T_{\text {inc }}(\lambda, \mu, h)=\exp \left[\frac{-\tau_{O 2, \text { total }}(\lambda, h)}{\mu}\right], \\
T_{\text {ref }}(\lambda, \mu, h)=\exp \left[-\tau_{O 2, \text { total }}(\lambda, h)\right] .
\end{gathered}
$$

The simulation is a bridge between observations and cloud properties. The observed radiance outside the oxygen A-band $\left(I^{0}\right)$ is suitable for retrieving the cloud optical depth because the observations are not disturbed by oxygen absorption $\left(T_{\mathrm{inc}}=T_{\text {ref }}=1\right)$.

$$
I^{0}\left(\lambda, \mu, \tau_{c}\right)=\int_{\lambda-\Delta \lambda}^{\lambda+\Delta \lambda} f\left(\lambda, \lambda^{\prime}\right) F_{\odot}\left(\lambda^{\prime}\right) \frac{\mu}{\pi} R\left(\mu, \tau_{c}, \omega=1\right) d \lambda^{\prime}
$$

Inside the oxygen A-band, the in-cloud absorption varies with the cloud geometrical properties (e.g., the cloud geometrical thickness). For evaluating the in-cloud absorption embodied in the parameterization, we considered a hypothetical case as a control, in which the cloud was seen as an opaque slab with a reflectance equal to $R\left(\mu, \tau_{c}, \omega=1\right)$. The radiance $I^{\prime}$ was an imaginary observation that was not affected by in-cloud absorption:

$$
I^{\prime}\left(\lambda, \mu, \tau_{c}, h, l\right)=\int_{\lambda-\Delta \lambda}^{\lambda+\Delta \lambda} f\left(\lambda, \lambda^{\prime}\right) F_{\odot}\left(\lambda^{\prime}\right) T_{\mathrm{inc}}\left(\lambda^{\prime}, \mu, h\right) \frac{\mu}{\pi} R\left(\mu, \tau_{c}, \omega=1\right) T_{\mathrm{ref}}\left(\lambda^{\prime}, h\right) d \lambda^{\prime}
$$

In Equation (40), cloud reflectance is the only difference from Equation (33). We used the radiance ratio $I / I^{\prime}$ as the quantification of the in-cloud absorption.

\section{Results}

\subsection{Contribution of Forward-Scattering}

We compared the new parameterization with the previous study [32] to verify the necessity of estimating forward-scattering impacts in $R_{\text {ph }}$. Figure 3 shows the truth reflectance (from DISORT) with two local peaks: $\theta<5^{\circ}$ and $\theta \approx 37^{\circ}$. The two local peaks correspond to the local peaks of phase function at the backward and the primary rainbow $\left(\vartheta \approx 143^{\circ}\right)$ which means that part of the reflectance is closely related to the phase function. This is why we kept the phase-function-dependent part in the parameterization for nadir observations. When we divide the reflection into single- and multi-scattering parts, the remaining local peaks in the multi-scattering curve (dashed line) indicate that, in addition to $R_{\mathrm{ss}}$, there are other parts related to the phase function. Instead, the improved formula cleanly extracts the phase-function-independent reflectance (dotted line), a curve with almost no peaks, fitting well with a simple rational polynomial (red cross). The vanishing peaks prove the necessity of quantifying the influence of forward-scattering on the reflectance.

\subsection{Improvements for Nonlinear Absorption}

Next, we tested the performance of higher-order terms in $S_{\mathrm{ms}}$, which is to approximate the nonlinear absorption attenuation in $R_{\infty}$,ms. Figure 4 a shows that the function $S_{\mathrm{ms}}$ decreases with increasing absorption and the declining rate varies with the single-scattering albedo. The variable rate suggests that the exponent in $S_{\mathrm{ms}}$ cannot be linear and the attenuation function $S$ in SACURA is a second-order function of $y$, which is not enough at $\omega<0.95$. Therefore, the hyperspectral application needs a high-order modification. Without the modification, the relative errors of the function $S$ are higher than 5\% when $\omega<0.95$, and exceed 20\% when $\omega<0.8$, as shown in Figure $4 \mathrm{~b}$. On the contrary, the relative errors of the modified function $S_{\mathrm{ms}}$ are less than $5 \%$ for $\omega>0.5$, as shown in Figure 4 a. 
The decline in the relative error demonstrates that the modified function $S_{\mathrm{ms}}$ is suitable for either weak or strong absorption cases.

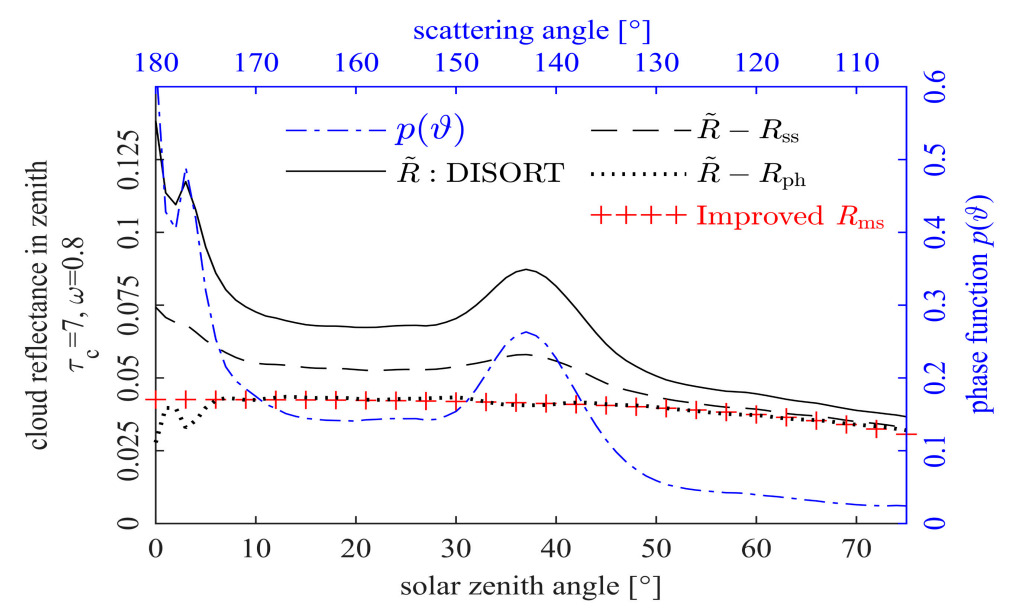

Figure 3. Cloud reflectance and its components. Dashed line: the reflection after removing the single-scattering part. Dotted line: similar to the dashed line, but further removes the contribution of forward-scattering.

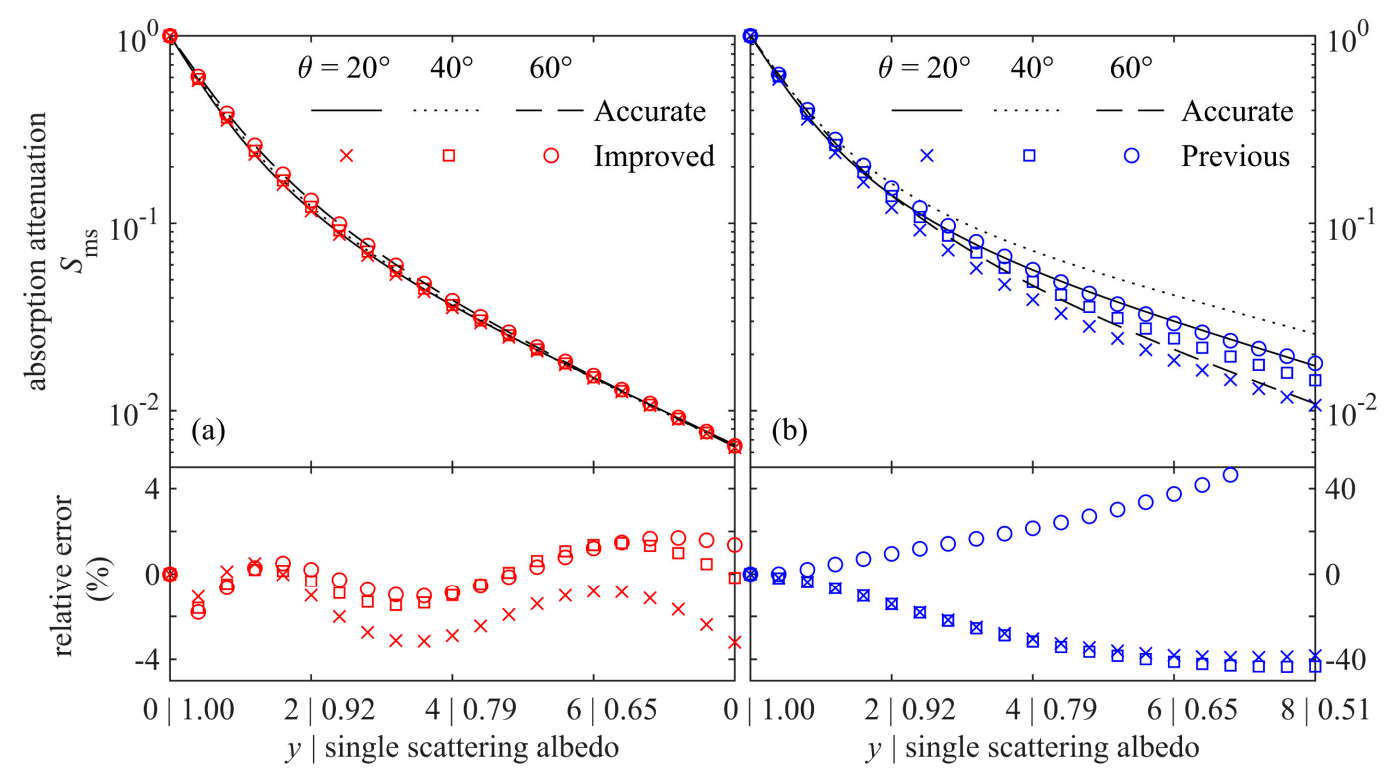

Figure 4. (a) The modified function $S_{\mathrm{ms}}$ and the relative errors compared to the accurate value from DISORT. The accurate $S_{\mathrm{ms}}$ is calculated as $\left(\widetilde{R}_{\infty}-R_{\infty, \mathrm{ph}}\right) /\left(\widetilde{R}_{\infty}^{0}-R_{\infty, \mathrm{ph}}^{0}\right)$, where $\widetilde{R}_{\infty}^{0}$ is the truth reflectance of the absorption-free semi-infinite cloud. (b) The function $S$ and the relative errors were calculated based on the previous study [32]. The accurate $S$ is calculated as $\widetilde{R}_{\infty} / \widetilde{R}_{\infty}^{0}$. Tests were at three solar zenith angles $(\theta)$.

Figure 5 is similar to Figure 4, but shows the performance of the modified function $H_{\mathrm{ms}}$. Without the modification, the relative errors of the function $H$ are higher than $10 \%$ when $\omega<0.95$ for $\theta=40^{\circ}$ and $\theta=60^{\circ}$, and exceed $25 \%$ when $\omega<0.8$ for all test cases, as shown in Figure $5 \mathrm{~b}$. With the modifications, the relative errors decrease to less than $5 \%$ when $\omega>0.95$ and less than $10 \%$ when $\omega>0.85$. 


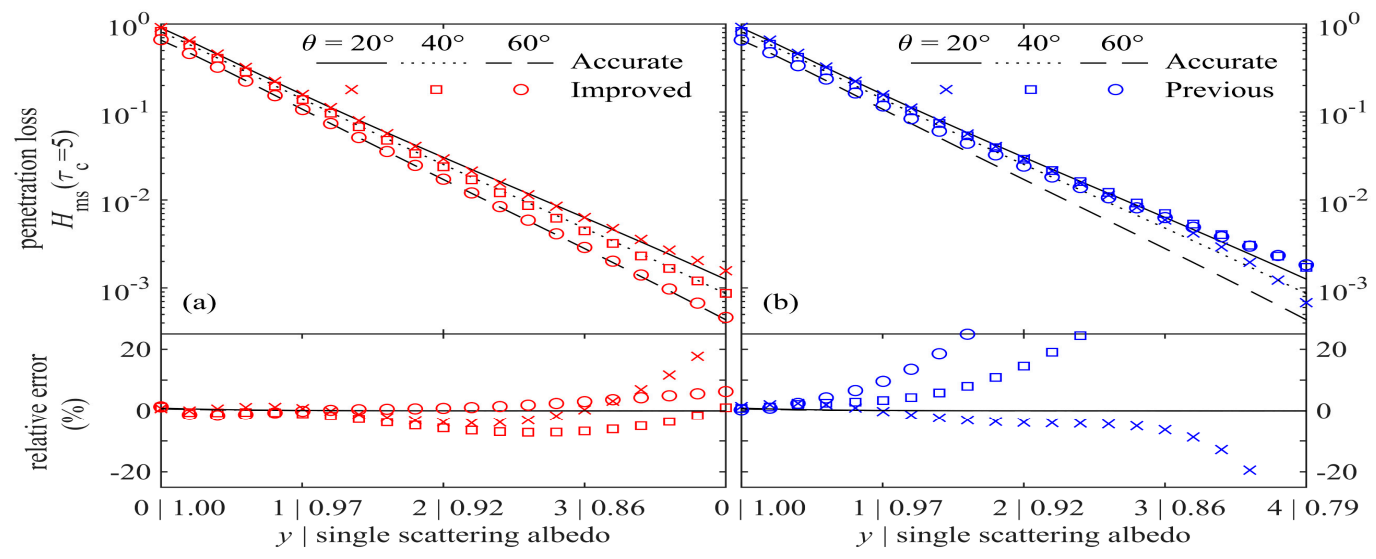

Figure 5. (a) The modified function $H_{\mathrm{ms}}$ and the relative errors compared to the accurate value from DISORT. The accurate $H_{\mathrm{ms}}$ is calculated as $\left(\widetilde{R}_{\infty}-R_{\infty, \mathrm{ph}}\right)-\left(\widetilde{R}-R_{\mathrm{ph}}\right)$. (b) The function $H$ and the relative errors were calculated based on the previous study [32]. The accurate $H$ is calculated as $\widetilde{R}_{\infty}-\widetilde{R}$. Tests were at three solar zenith angles $(\theta)$.

\subsection{Errors in Parameterization}

Figure 6 comprehensively shows the calculated cloud reflectance and relative errors. The parameterization performs better on optically thick clouds than thin clouds. The modifications in $R_{\text {ph }}$ make the approximations match the truth at $5^{\circ} \leq \theta \leq 75^{\circ}$, including the local peaks, even when applied to the optically relatively thin cloud. For cases with strong absorption, the modifications in $S_{\mathrm{ms}}$ and $H_{\mathrm{ms}}$ keep the relative error $\delta_{2}$ within $5 \%$. Figure 6 shows the usability of the improved physical parameterization in cases with different solar zenith angles, cloud optical depths, and single-scattering albedos.

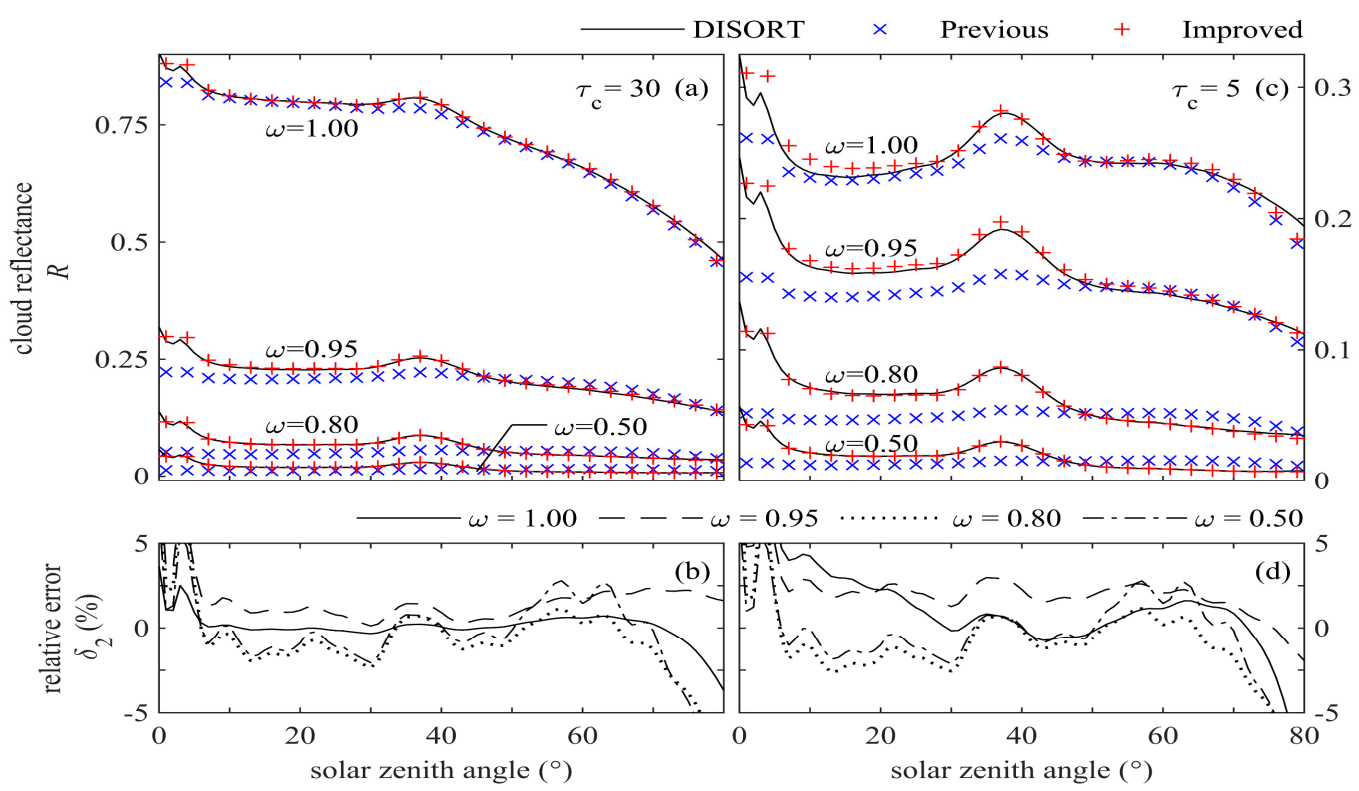

Figure 6. The (a) reflectance and (b) relative error of the parameterization in cases with different single-scattering albedos $(\omega)$ and solar zenith angles, compared with the previous study [32] and DISORT. (c,d) Similar to Figure 4a,b, but for optically thinner clouds. 


\subsection{Accuracy Analysis Outside the Oxygen A-Band}

The parameterization is to simulate the hyperspectral nadir observation in the oxygen A-band rapidly, and Figure 7 shows the process. In Figure $7 \mathrm{~b}$, the improved parameterization gives a set of cloud reflectance, which is close to the precise value from DISORT. The simulated TOA radiance, as shown in Figure 7c is a combination of the solar radiation, as shown in Figure 7a, the transmittivity above the cloud, the cloud reflectance, and the instrument response function.

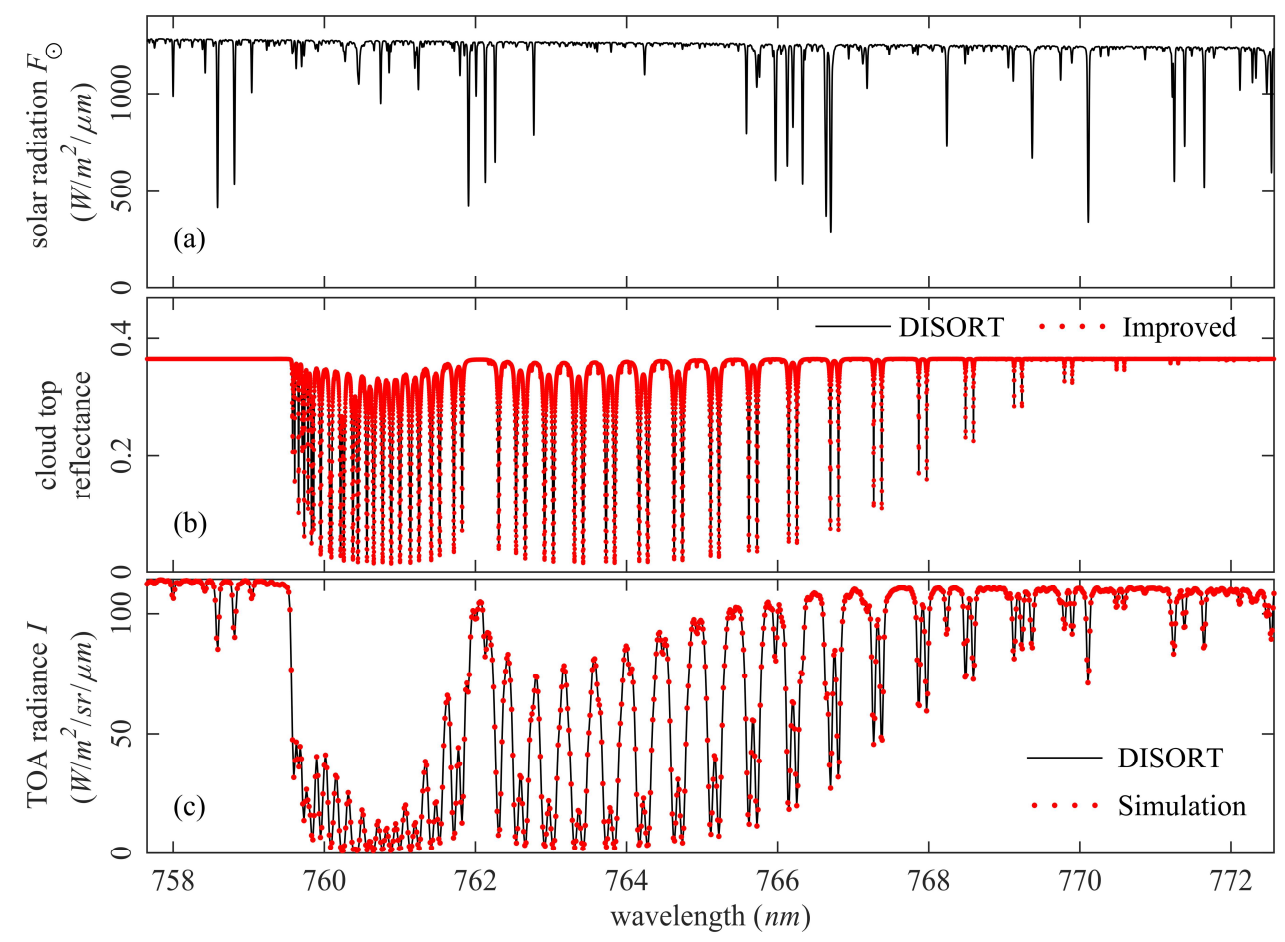

Figure 7. An example of hyperspectral simulations at the top of the atmosphere. (a) Solar radiation at the top of the atmosphere. (b) The cloud reflectance calculated by the physical parameterization and compared with the precise reflectance from DISORT. (c) The simulated hyperspectral radiance at the top of the atmosphere. The cloud optical depth is 7, the cloud-top height is $1.25 \mathrm{~km}$, the cloud geometrical thickness is $0.5 \mathrm{~km}$, and the solar zenith angle is $40^{\circ}$.

The radiation outside the band is absorption-free and suitable for retrievals of cloud optical depth. Thus, it is necessary to test the accuracy of the simulated radiance outside oxygen A band. Figure $8 \mathrm{a}$ shows a series of radiances calculated by the physical parameterization corresponding to different cloud optical depths. The radiance changes significantly in some channels, including channels located near $759.3 \mathrm{~nm}$ and $771 \mathrm{~nm}$ outside the oxygen A-band, and some non-absorption channels located inside the band that can only be observed by hyperspectral sensors (e.g., channels near $767.6 \mathrm{~nm}$ ). Figure $8 \mathbf{b}$ shows the relative errors of the hyperspectral simulations, and at least half of the samples have a relative error of less than $0.5 \%$ on the channels mentioned above. The relative error increases to about $4 \%$ if all the samples are counted. Due to the modification for enhanced absorption, the simulated radiance also has a high accuracy of about $2 \%$ in the absorption channels, even in channels containing oxygen absorption lines. 


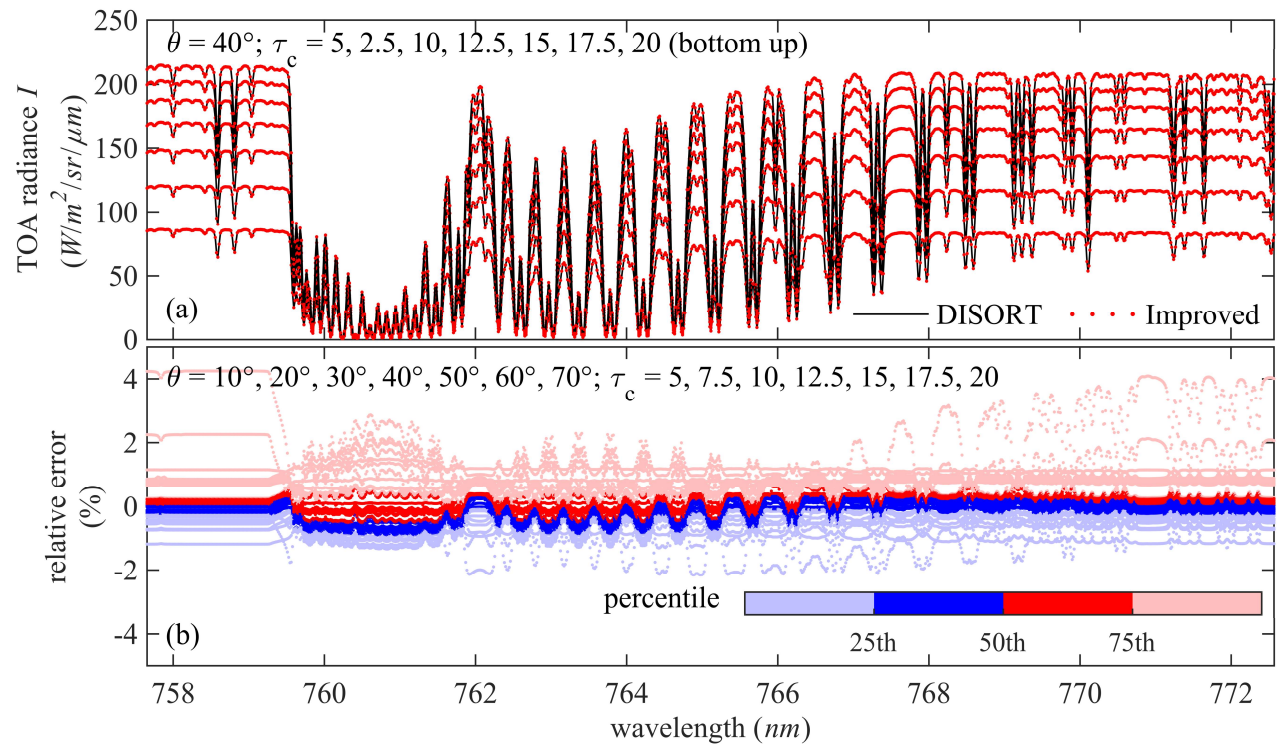

Figure 8. A series of TOA radiances, simulated at different cloud optical depths compared with the precise radiance derived from DISORT. (a) The radiance outside the oxygen A-band increases with the thickening cloud optical depth. (b) The relative error of the simulation outside the oxygen A-band. Each channel has 49 samples to estimate percentiles.

\subsection{Accuracy Analysis Inside the Oxygen A-Band}

The absorption in the oxygen A-band is the basis of the cloud structure retrievals. The parameterization considers the influence of in-cloud absorption and is established for retrievals of cloud geometrical thickness. We used the radiance ratio to quantify the in-cloud absorption. The numerator radiance was simulated as the process in Figure 7, and the denominator radiance was simulated under the opaque slab assumption, as shown in Figure 9. The radiance ratio is less than 1 in the absorption channel—the stronger the absorption, the smaller the ratio.

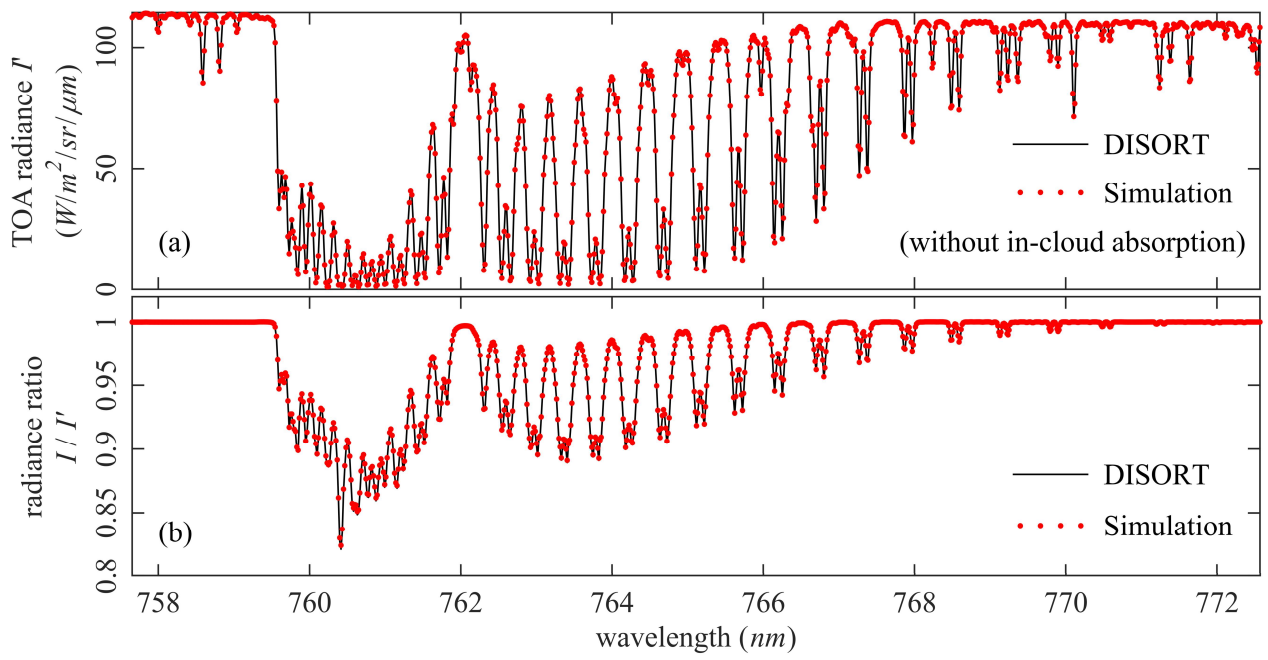

Figure 9. An example of the hyperspectral radiance ratio, the quantification of the in-cloud absorption. (a) The hyperspectral radiance of the hypothetical case, in which the cloud is an opaque plate. The simulated radiance is compared with the precise simulation based on DISORT. (b) The simulated hyperspectral radiance ratio. The cloud optical properties are the same as in Figure 8.

Figure 10a shows a series of radiance ratios, which decrease gradually in the absorption channels as the cloud geometrically thickens. The change is most apparent near the oxygen absorption lines, 
such as $760.6 \mathrm{~nm}$ and $763.8 \mathrm{~nm}$. Figure $10 \mathrm{~b}$ shows the relative error of the simulated radiance ratio. At least half of the samples have a relative error of less than $1 \%$, and the relative errors of all samples are about $4 \%$ on the absorption channels.

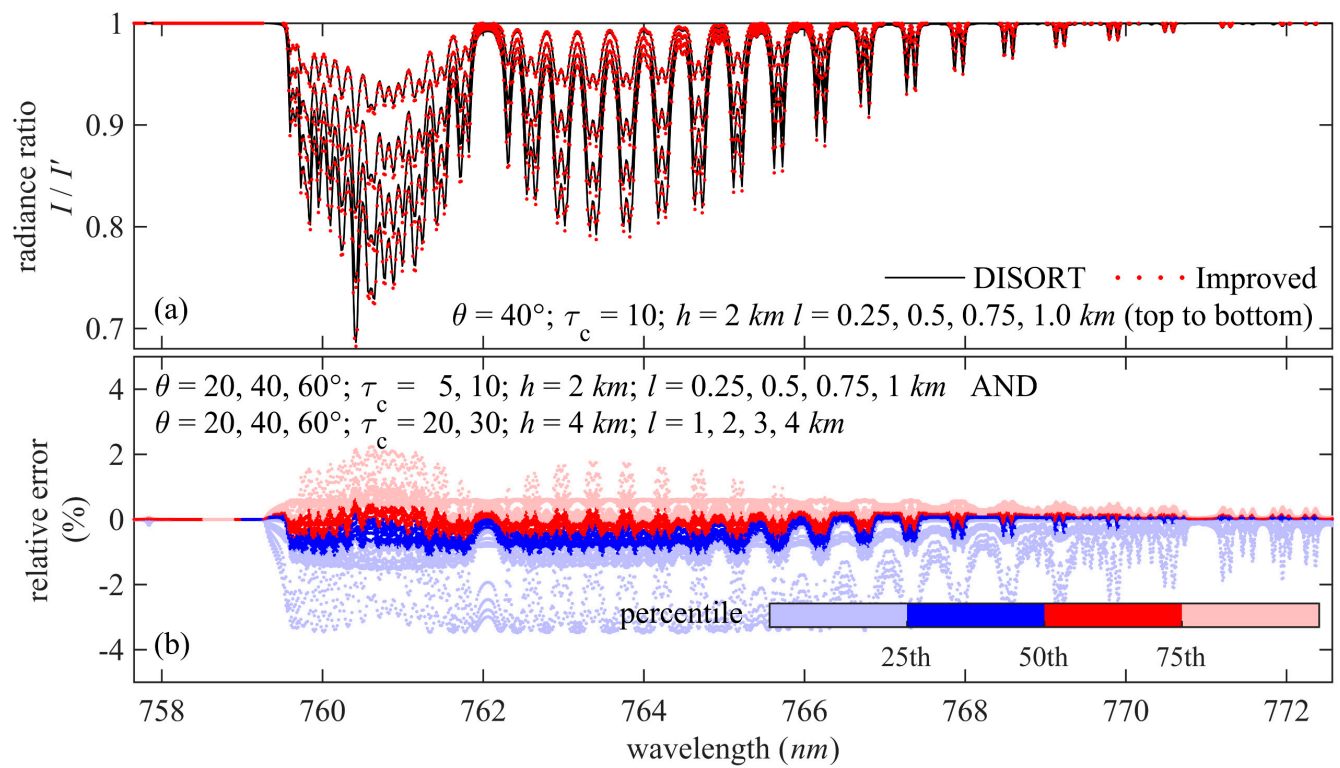

Figure 10. Comparison of the simulated radiance ratio from the parameterization and DISORT at different cloud geometrical thicknesses. (a) The radiance ratio inside the oxygen A-band decreases with the thinning cloud geometrical thickness. (b) The relative error of the radiance ratio inside the oxygen A-band. The percentiles are estimated from 24 thin clouds and 24 thick clouds.

\section{Discussion and Conclusions}

The accurate parametric cloud reflectance is the premise of cloud retrieval. The parameterization developed for space-borne moderate spectral resolution observations should not be used to calculate the hyperspectral reflectance directly. The hyperspectral measurements in the oxygen A-band have several strong absorption channels where the in-cloud scattering and absorption are different from those in weak absorption channels. We show that the proportion of single-scattering in the reflection increases gradually with the absorption enhancement and the influence of cloud droplets forward-scattering is important for the optically thin cloud. The single-scattering represents the radiative transfer dependent on the phase function, while the multi-scattering represents the radiative transfer independent on the phase function. The two scatterings are different, and it is necessary to establish formulas for each of them to parameterize their contribution to the cloud-top reflectance.

In this study, we developed a fast physical parameterization method for cloud reflectance in the oxygen A-band for hyperspectral remote sensing. Firstly, we quantified the influences of forward-scattering on reflectance. Secondly, the nonlinear absorption was parameterized, and the modified formulas were applicable to both strong absorption lines and weak absorption bands. After integrating all the improvements, the relative error of the parameterization was less than $5 \%$ when $5^{\circ} \leq \theta \leq 75^{\circ}$ compared with the precise value from DISORT (Discrete Ordinates Radiative Transfer Program Multi-Layered Plane-Parallel Medium), even for the optically relatively thin clouds (the cloud optical depth is higher than 5) or strong absorption lines (the single-scattering albedo is lower than 0.95 ). For optically thick clouds, the relative error reduced to $2 \%$. The performance of the parameterization in the hyperspectral simulation was estimated, too. The simulation was carried out in a simplified atmospheric model with the focus on the accuracy in calculating the observed cloud reflection, which ignores the molecular scattering, aerosol scattering, and reflection from the underlying surface. The relative errors of all test cases were less than $5 \%$ for the radiance outside the oxygen A-band that can be used for cloud optical depth retrievals, and at least half of the test cases had 
a relative error of less than $0.5 \%$. The relative errors in all test cases were less than $4 \%$ for the radiance ratio in the oxygen A-band related to the cloud geometrical thickness, and at least half of the test cases had a relative error of less than $1 \%$.

Generally, the accuracy of the parameterized cloud reflectance has been improved, but there are still cases whose relative error is a little bit higher than others (e.g., the cases at $\theta<5^{\circ}$ ), as shown in Figures 3 and 6. Compared with the slant incidence case, the near-vertical transfer would encounter fewer cloud droplets and increase the proportion of reflections that experience only a few scatterings. Optically thin clouds are in a similar situation; e.g., the larger relative error in Figure $6 \mathrm{~d}$ than that in Figure $6 \mathrm{~b})$. Although we have introduced the correction $\Delta t$ for these reflections, their parameterization is still incomplete. There is another error at $\theta>75^{\circ}$ in Figure 6, mainly from the error of the escape function $K$ at large angles [37], but the shadows caused by large solar zenith angles are more severe than the error of the parameterization.

This paper is an extension of the previous work and can replace the part of SACURA that calculates the cloud reflectance without interfering with other parts that deal with aerosol scattering and underlying surface reflection. It helps improve retrieval efficiency based on hyperspectral observations and provides cloud geometric thickness products. On the other hand, more details, such as polarized radiative transfer, multidirectional measurements, and cloud droplet effective radius, need to be considered before applying the parameterization widely. For example, on OCO-2 (Orbiting Carbon Observatory-2), a linear polarizer is installed to reduce the stray light [45]. If the parameterization is to be applied to OCO-2 observations, it is necessary to modify the parameterization to adapt to the polarized radiative transfer. Several studies suggest that the variability in angular measurements is sensitive to the cloud geometrical thickness [46,47], and the parameterization can also be extended to these studies if the observation zenith angle is added to the input of the parameterization. Besides, the influence of the cloud effective radius on cloud scattering is also unresolved, which is related to both phase function and cloud droplet number density. At present, the $\mathrm{C} 1$ phase function is used in the parameterization, which can potentially be biased and not applicable to a wide variety of clouds. It may improve the parameterization's applicability in cloud retrievals that introduce the cloud droplet effective radius into the parameterization. It is also necessary to detect and quantify other clouds, such as the water cloud covered by a thin ice cloud [48] or the profile of an optical thin cloud [49].

The cloud reflectance parameterization lays the foundation for the future rapid retrievals of marine water clouds, especially for the cloud geometric thickness of clouds. In the future, we will use OCO-2 oxygen A-band observations, based on the rapid algorithm, to obtain the cloud optical depth, cloud top height, and cloud geometric thickness, taking into account the instrument characteristics, such as polarization and instrument characteristics, including the observation noise and the wavelength registration error [50], as well as the atmospheric model containing aerosol scattering and surface reflection. Although the cloud retrieval algorithm has been studied based on OCO-2 observations, the cloud products retrieved by the rapid algorithm are not yet available. The rapid retrieval usually comes at the cost of accuracy and the unknown quality of cloud products obtained by the rapid retrieval algorithm is the topic for our future research.

Author Contributions: Conceptualization, J.Y. and S.L. (Siwei Li); methodology, J.Y.; software, J.Y.; validation, J.Y.; formal analysis, J.Y.; investigation, L.Z. and S.L. (Sheng Liu); resources, L.Z. and S.L. (Sheng Liu); data curation, L.Z. and S.L. (Sheng Liu); writing—original draft preparation, J.Y.; writing—review and editing, J.Y., S.L. (Siwei Li), F.M., Q.M. and W.G.; visualization, J.Y.; supervision, S.L. (Siwei Li); project administration, S.L. (Siwei Li); funding acquisition, S.L. (Siwei Li). All authors have read and agreed to the published version of the manuscript.

Funding: This research was funded by the National Natural Science Foundation of China, grant number 41975022.

Acknowledgments: We are grateful to the Atmospheric and Environmental Research (AER) for providing the code of the Line-By-Line Radiative Transfer Model and the AER solar irradiance model. We are also grateful to Warren Wiscombe, Si-Chee Tsay, Istvan Laszlo, Knut Stamnes, Snorre Stamnes, Zhonghai Jin, and Zhenyi Lin for their working on the development of the Discrete Ordinates Radiative Transfer Program Multi-Layered Plane-Parallel Medium. 
Conflicts of Interest: The authors declare no conflict of interest.

\section{References}

1. Yamamoto, G.; Wark, D. Discussion of the letter by RA Hanel,“Determination of cloud altitude from a satellite". J. Geophys. Res. 1961, 66, 3596. [CrossRef]

2. Wu, M.L.C. Remote-Sensing of Cloud-Top Pressure Using Reflected Solar-Radiation in the Oxygen a-Band. J. Clim. Appl. Meteorol. 1985, 24, 539-546. [CrossRef]

3. Koelemeijer, R.B.A.; Stammes, P.; Hovenier, J.W.; de Haan, J.F. A fast method for retrieval of cloud parameters using oxygen A band measurements from the Global Ozone Monitoring Experiment. J. Geophys. Res.-Atmos. 2001, 106, 3475-3490. [CrossRef]

4. Rozanov, V.V.; Kokhanovsky, A.A. Semianalytical cloud retrieval algorithm as applied to the cloud top altitude and the cloud geometrical thickness determination from top-of-atmosphere reflectance measurements in the oxygen A band. J. Geophys. Res.-Atmos. 2004, 109, D05202. [CrossRef]

5. Rodriguez, D.G.L.; Thomas, W.; Livschitz, Y.; Ruppert, T.; Albert, P.; Hollmann, R. Cloud properties derived from GOME/ERS-2 backscatter data for trace gas retrieval. IEEE Trans. Geosci. Remote Sens. 2007, 45, 2747-2758. [CrossRef]

6. Loyola, D.G.; Gimeno García, S.; Lutz, R.; Argyrouli, A.; Romahn, F.; Spurr, R.J.D.; Pedergnana, M.; Doicu, A.; Molina García, V.; Schüssler, O. The operational cloud retrieval algorithms from TROPOMI on board Sentinel-5 Precursor. Atmos. Meas. Tech. 2018, 11, 409-427. [CrossRef]

7. Burrows, J.P.; Weber, M.; Buchwitz, M.; Rozanov, V.; Ladstätter-Weißenmayer, A.; Richter, A.; DeBeek, R.; Hoogen, R.; Bramstedt, K.; Eichmann, K.-U.; et al. The Global Ozone Monitoring Experiment (GOME): Mission Concept and First Scientific Results. J. Atmos. Sci. 1999, 56, 151-175. [CrossRef]

8. Bovensmann, H.; Burrows, J.P.; Buchwitz, M.; Frerick, J.; Noel, S.; Rozanov, V.V.; Chance, K.V.; Goede, A.P.H. SCIAMACHY: Mission objectives and measurement modes. J. Atmos. Sci. 1999, 56, 127-150. [CrossRef]

9. Kokhanovsky, A.A.; Rozanov, V.V.; von Hoyningen-Huene, W.; Bovensmann, H.; Burrows, J.P.; Baltink, H.K. The Determination of Cloud Altitudes Using SCIAMACHY Onboard ENVISAT. IEEE Geosci. Remote Sens. Lett. 2004, 1, 211-214. [CrossRef]

10. Kokhanovsky, A.; Vountas, M.; Burrows, J.P. Global Distribution of Cloud Top Height as Retrieved from SCIAMACHY Onboard ENVISAT Spaceborne Observations. Remote Sens. 2011, 3, 836-844. [CrossRef]

11. Munro, R.; Lang, R.; Klaes, D.; Poli, G.; Eisinger, M. The GOME-2 instrument on the Metop series of satellites: Instrument design, calibration, and level 1 data processing-An overview. Atmos. Meas. Tech. 2015, 9, 1279-1301. [CrossRef]

12. Lelli, L.; Kokhanovsky, A.A.; Rozanov, V.V.; Vountas, M.; Sayer, A.M.; Burrows, J.P. Seven years of global retrieval of cloud properties using space-borne data of GOME. Atmos. Meas. Tech. 2012, 5, 1551-1570. [CrossRef]

13. Wang, P.; Stammes, P. Evaluation of SCIAMACHY Oxygen A band cloud heights using Cloudnet measurements. Atmos. Meas. Tech. 2014, 7, 1331-1350. [CrossRef]

14. Lelli, L.; Kokhanovsky, A.A.; Rozanov, V.V.; Vountas, M.; Burrows, J.P. Linear trends in cloud top height from passive observations in the oxygen A-band. Atmos. Chem. Phys. 2014, 14, 5679-5692. [CrossRef]

15. Lelli, L.; Weber, M.; Burrows, J.P. Evaluation of SCIAMACHY ESA/DLR Cloud Parameters Version 5.02 by Comparisons to Ground-Based and Other Satellite Data. Front. Environ. Sci. 2016, 4, 43. [CrossRef]

16. Rozanov, V.V.; Kokhanovsky, A.A.; Burrows, J.P. The determination of cloud altitudes using GOME reflectance spectra: Multilayered cloud systems. IEEE Trans. Geosci. Remote Sens. 2004, 42, 1009-1017. [CrossRef]

17. Koelemeijer, R.B.A.; Stammes, P.; Hovenier, J.W.; de Haan, J.F. Global distributions of effective cloud fraction and cloud top pressure derived from oxygen A band spectra measured by the Global Ozone Monitoring Experiment: Comparison to ISCCP data. J. Geophys. Res.-Atmos. 2002, 107, AAC 5-1-AAC 5-9. [CrossRef]

18. Vanbauce, C.; Cadet, B.; Marchand, R.T. Comparison of POLDER apparent and corrected oxygen pressure to ARM/MMCR cloud boundary pressures. Geophys. Res. Lett. 2003, 30, 1212-1215. [CrossRef]

19. Schuessler, O.; Rodriguez, D.G.L.; Doicu, A.; Spurr, R. Information Content in the Oxygen A-Band for the Retrieval of Macrophysical Cloud Parameters. IEEE Trans. Geosci. Remote Sens. 2014, 52, 3246-3255. [CrossRef] 
20. Rozanov, V.V.; Kokhanovsky, A.A. Determination of Cloud Geometrical Thickness Using Backscattered Solar Light in a Gaseous Absorption Band. IEEE Geosci. Remote Sens. Lett. 2006, 3, 250-253. [CrossRef]

21. Obrien, D.M.; Mitchell, R.M. Error-Estimates for Retrieval of Cloud-Top Pressure Using Absorption in the a-Band of Oxygen. J. Appl. Meteorol. 1992, 31, 1179-1192. [CrossRef]

22. Heidinger, A.K.; Stephens, G.L. Molecular line absorption in a scattering atmosphere. Part II: Application to remote sensing in the O2 A band. J. Atmos. Sci. 2000, 57, 1615-1634. [CrossRef]

23. Hamazaki, T.; Kaneko, Y.; Kuze, A.; Kondo, K. Fourier transform spectrometer for Greenhouse gases Observing Satellite (GOSAT). In Enabling Sensor and Platform Technologies for Spaceborne Remote Sensing; Komar, G.J., Wang, J., Kimura, T., Eds.; Spie-Int Soc Optical Engineering: Bellingham, WA, USA, 2005; Volume 5659, pp. 73-80.

24. Frankenberg, C.; Pollock, R.; Lee, R.A.M.; Rosenberg, R.; Blavier, J.F.; Crisp, D.; O’Dell, C.W.; Osterman, G.B.; Roehl, C.; Wennberg, P.O.; et al. The Orbiting Carbon Observatory (OCO-2): Spectrometer performance evaluation using pre-launch direct sun measurements. Atmos. Meas. Tech. 2015, 8, 301-313. [CrossRef]

25. Richardson, M.; Stephens, G.L. Information content of OCO-2 oxygen A-band channels for retrieving marine liquid cloud properties. Atmos. Meas. Tech. 2018, 11, 1515-1528. [CrossRef]

26. Richardson, M.; Leinonen, J.; Cronk, H.Q.; McDuffie, J.; Lebsock, M.D.; Stephens, G.L. Marine liquid cloud geometric thickness retrieved from OCO-2's oxygen A-band spectrometer. Atmos. Meas. Tech. 2019, 12, 1717-1737. [CrossRef]

27. Reuter, M.; Buchwitz, M.; Schneising, O.; Noël, S.; Rozanov, V.; Bovensmann, H.; Burrows, J. A Fast Atmospheric Trace Gas Retrieval for Hyperspectral Instruments Approximating Multiple Scattering-Part 1: Radiative Transfer and a Potential OCO-2 XCO2 Retrieval Setup. Remote Sens. 2017, 9, 1159. [CrossRef]

28. Del Águila, A.; Efremenko, D.S.; Molina García, V.; Kataev, M.Y. Cluster Low-Streams Regression Method for Hyperspectral Radiative Transfer Computations: Cases of $\mathrm{O}_{2} \mathrm{~A}$ - and $\mathrm{CO}_{2}$ Bands. Remote Sens. 2020, 12, 1250. [CrossRef]

29. Kokhanovsky, A.A.; Rozanov, V.V.; Burrows, J.P.; Eichmann, K.U.; Lotz, W.; Vountas, M. The SCIAMACHY cloud products: Algorithms and examples from ENVISAT. In Atmospheric Remote Sensing: Earth's Surface, Troposphere, Stratosphere and Mesosphere-I; Burrows, J.P., Eichmann, K.U., Eds.; Elsevier Science Ltd.: Oxford, UK, 2005; Volume 36, pp. 789-799.

30. Kokhanovsky, A.A.; Rozanov, V.V. The reflection function of optically thick weakly absorbing turbid layers: A simple approximation. J. Quant. Spectrosc. Radiat. 2003, 77, 165-175. [CrossRef]

31. Nauss, T.; Kokhanovsky, A.A. Retrieval of warm cloud optical properties using simple approximations. Remote Sens. Environ. 2011, 115, 1317-1325. [CrossRef]

32. Kokhanovsky, A.A.; Rozanov, V.V. The physical parameterization of the top-of-atmosphere reflection function for a cloudy atmosphere-Underlying surface system: The oxygen A-band case study. J. Quant. Spectrosc. Radiat. 2004, 85, 35-55. [CrossRef]

33. Kokhanovsky, A.A. Reflection of light from nonasbsorbing semi-infinite cloudy media: A simple approximation. J. Quant. Spectrosc. Radiat. 2004, 85, 25-33. [CrossRef]

34. Kokhanovsky, A.A. Simple approximate formula for the reflection function of a homogeneous, semi-infinite turbid medium. J. Opt. Soc. Am. A Opt. Image Sci. Vis. 2002, 19, 957-960. [CrossRef] [PubMed]

35. Kokhanovsky, A.A. The accuracy of selected approximations for the reflection function of a semi-infinite turbid medium. J. Phys. D Appl. Phys. 2002, 35, 1057-1062. [CrossRef]

36. Kokhanovsky, A.A. Optical properties of semiinfinite turbid media: Some simple analytical approximations. Opt. Eng. 2003, 42, 2040-2046. [CrossRef]

37. Kokhanovsky, A.A.; Rozanov, V.V.; Zege, E.P.; Bovensmann, H.; Burrows, J.P. A semianalytical cloud retrieval algorithm using backscattered radiation in 0.4-2.4 $\mu \mathrm{m}$ spectral region. J. Geophys. Res.-Atmos. 2003, 108, AAC 4-1-AAC 4-19. [CrossRef]

38. Kokhanovsky, A.A.; Nakajima, T.; Zege, E.P. Physically based parameterizations of the short-wave radiative characteristics of weakly absorbing optically thick media: Application to liquid-water clouds. Appl. Opt. 1998, 37, 4750-4757. [CrossRef]

39. Hahn, C.J.; Rossow, W.B.; Warren, S.G. ISCCP Cloud Properties Associated with Standard Cloud Types Identified in Individual Surface Observations. J. Clim. 2001, 14, 11-28. [CrossRef] 
40. Stamnes, K.; Tsay, S.C.; Wiscombe, W.; Jayaweera, K. Numerically stable algorithm for discrete-ordinate-method radiative transfer in multiple scattering and emitting layered media. Appl. Opt. 1988, 27, 2502-2509. [CrossRef]

41. Boesch, H.; Brown, L.; Castano, R.; Christi, M.; Crisp, D.; Eldering, A.; Fisher, B.; Frankenberg, C.; Gunson, M.; Granat, R. Orbiting Carbon Observatory (OCO)-2 Level 2 Full Physics Algorithm Theoretical Basis Document. Available online: https://docserver.gesdisc.eosdis.nasa.gov/public/project/OCO/OCO2_L2_ATBD.V8.pdf (accessed on 12 June 2020).

42. Lee, R.A.M.; O’Dell, C.W.; Wunch, D.; Roehl, C.M.; Osterman, G.B.; Blavier, J.F.; Rosenberg, R.; Chapsky, L.; Frankenberg, C.; Hunyadi-Lay, S.L.; et al. Preflight Spectral Calibration of the Orbiting Carbon Observatory 2. IEEE Trans. Geosci. Remote Sens. 2017, 55, 2499-2508. [CrossRef]

43. Coddington, O.; Lean, J.L.; Pilewskie, P.; Snow, M.; Lindholm, D. A Solar Irradiance Climate Data Record. Bull. Am. Meteorol. Soc. 2016, 97, 1265-1282. [CrossRef]

44. Clough, S.A.; Shephard, M.W.; Mlawer, E.J.; Delamere, J.S.; Iacono, M.J.; Cady-Pereira, K.; Boukabara, S.; Brown, P.D. Atmospheric radiative transfer modeling: A summary of the AER codes. J. Quant. Spectrosc Radiat. 2005, 91, 233-244. [CrossRef]

45. Rosenberg, R.; Maxwell, S.; Johnson, B.C.; Chapsky, L.; Lee, R.A.M.; Pollock, R. Preflight Radiometric Calibration of Orbiting Carbon Observatory 2. IEEE Trans. Geosci. Remote Sens. 2017, 55, 1994-2006. [CrossRef]

46. Ferlay, N.; Thieuleux, F.; Cornet, C.; Davis, A.B.; Dubuisson, P.; Ducos, F.; Parol, F.; Riedi, J.; Vanbauce, C. Toward New Inferences about Cloud Structures from Multidirectional Measurements in the Oxygen A Band: Middle-of-Cloud Pressure and Cloud Geometrical Thickness from POLDER-3/PARASOL. J. Appl. Meteorol. Climatol. 2010, 49, 2492-2507. [CrossRef]

47. Merlin, G.; Riedi, J.; Labonnote, L.C.; Cornet, C.; Davis, A.B.; Dubuisson, P.; Desmons, M.; Ferlay, N.; Parol, F. Cloud information content analysis of multi-angular measurements in the oxygen A-band: Application to 3MI and MSPI. Atmos. Meas. Tech. 2016, 9, 4977-4995. [CrossRef]

48. Li, S.; Min, Q. Diagnosis of multilayer clouds using photon path length distributions. J. Geophys. Res. 2010, 115. [CrossRef]

49. Li, S.; Min, Q. Retrievals of vertical profiles of stratus cloud properties from combined oxygen A-band and radar observations. J. Geophys. Res. Atmos. 2013, 118, 769-778. [CrossRef]

50. Li, S.; Min, Q. Wavelength registration of high resolution oxygen A-band spectral measurements. J. Quant. Spectrosc Radiat. 2013, 122, 106-113. [CrossRef] 